\title{
Rehabilitación de la filosofía práctica y neo-aristotelismo
}

The rehabilitation of practical philosophy and neo-Aristotelianism

Franco Volpi $(\dagger)$

Università di Padova

Abstract: This article describes the German debate concerning the "rehabilitation of practical philosophy," locates its origin in neo-Aristotelian thought, and formulates three neo-Aristotelian theses derived from the Aristotelian understanding of praxis. Neo-Aristotelianism is shown to be a response to the standard modern "scientific" understanding of practical knowledge, which paradoxically entailed a regression in the understanding of practical knowledge (and of the relation between theory and praxis), and which led to fundamental problems in the development of modern sciences of action.

Keywords: Neo-Aristotelianism, practical rationality, theory, praxis, phronesis.
Resumen: En este trabajo se describe el debate que tuvo lugar en Alemania sobre "la rehabilitación de la filosofía práctica", se localiza su origen en las posiciones filosóficas "neo-aristotélicas", y se formulan tres tesis fundamentales que los neo-aristotélicos retoman a partir de la comprensión aristotélica de la praxis. Se muestra el neo-aristotelismo como una reacción frente a la comprensión "científica" del saber práctico típica de la modernidad, que supuso, paradójicamente, una regresión en la comprensión del saber práctico (y de la relación entre la teoría y la praxis), de la que nacieron los problemas fundamentales de la determinación moderna de las ciencias del obrar.

Palabras clave: Neo-aristotelismo, racionalidad práctica, teoría, praxis, frónesis.

Publicado originalmente en francés como Réhabilitation de la philosophie pratique et néo-aristotélisme, en P. Aubenque, A. TORDEsIllas (eds.), Aristote politique. Études sur la Politique d'Aristote (PUF, Paris, 1993) 461-484.

Traducido al español por AlejAndRo G. VIGo para “Anuario Filosófico" XXXII/1 (1999) 315-342. Publicado posteriormente en inglés como The rehabilitation of practical philosophy and neo-Aristotelianism, en R. C. BARTLET, S. D. ColuIns (eds.), Action and contemplation: studies in the moral and political thought of Aristotle (State University of New York Press, Albany, 1999) 3-25.

DOI: $10.15581 / 009.50 .1 .189-214$ 


\section{El Debate ALEMÁN \\ SOBRE LA 'FILOSOFÍA PRÁCTICA'}

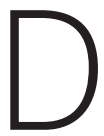

esde el comienzo de los años sesenta y hasta el fin de los años setenta del siglo XX tuvo lugar en Alemania un debate intenso, que se hizo famoso bajo el título de "rehabilitación de la filosofía práctica". Desde un punto de vista general, se pueden distinguir dos momentos principales en el origen y desarrollo de este debate: el primer momento - preparado por los escritos de algunos filósofos políticos de origen alemán, emigrados a los Estados Unidos, como Leo Strauss, Eric Voegelin y Hannah Arendtllega a la maduración en los años sesenta y se caracteriza principalmente por el redescubrimiento de la actualidad de los modelos de filosofía práctica de Aristóteles, y luego de Kant, así como por la tentativa de referirse a dichos modelos para proponer una nueva consideración filosófica del dominio del obrar; el segundo momento ha tenido, sobre todo, un carácter teórico-sistemático, en el cual los problemas relevados por la nueva proposición de los modelos aristotélicos y kantianos fueron abordados y tratados en el cuadro de una discusión más general, en la cual tomaron la palabra las principales escuelas filosóficas alemanas contemporáneas, como la antigua Escuela de Frankfurt, de inspiración hegeliano-marxista (Theodor Wiesengrund, Adorno y el joven Jürgen Habermas), el racionalismo crítico (Hans Albert, Hans Lenk y, bajo cierto aspecto, Ernst Topitsch), la hermenéutica filosófica de Hans-Georg Gadamer y su discípulo Rüdiger Bubner, pero también Joachim Ritter y su escuela (Hermann Lübbe, Odo Marquard, Willi Oelmüller, Günter Bien, Reinhart Maurer), el constructivismo de la Escuela de Erlangen y de Constanza (fundada por Paul Lorenzen, desarrollada por Friedrich

1. La primera documentación general sobre este debate fue recopilada por M. RIEDEL (ed.), Rebabilitierung der praktischen Philosophie, 2 vols. (Rombach, Freiburg i. Br., 1972-1974). Para una reconstrucción de conjunto del debate, me permito remitir a mi artículo: $L a$ rinascita della filosofía pratica in Germania, en C. Pacchiani (ed.), Filosofía pratica e scienza política (Francisci, Abano Terme-Padova, 1980) 11- 97; para un análisis de ciertos aspectos particulares, cf. A. DA RE, L'etica tra felicità e dovere. L'attuale dibattito sulla filosofia pratica (Dehoniane, Bologna, 1986); L. ConTella, Aristotele e la razionalità della prassi. Una analisi del dibattito sulla filosofia pratica aristotelica in Germania (Jouvence, Roma, 1987). 
Kambartel y Oswald Schwemmer, pero luego disgregada) y, por último, la nueva Escuela de Frankfurt de Karl-Otto Apel y del último Habermas, con su proyecto de una ética del discurso fundada en una pragmática transcendental o universal. En la discusión compleja que derivó intervinieron, en mayor o menor medida, los representantes más prestigiosos y más renombrados del pensamiento filosófico alemán contemporáneo; esta discusión implicó y motivó asimismo la toma de posición por parte de pensadores activos en campos disciplinarios no propiamente filosóficos, como por ejemplo el sociólogo Niklas Luhmann (y su crítica de la obsolescencia de la categoría de "fin")2, el etólogo Konrad Lorenz (sobre todo, en su discusión de la génesis biológica de las conductas morales, en tanto residuo de reglas instintivas) ${ }^{3}$ o incluso el antropólogo Arnold Gehlen (por su fundación antropo-biológica de las instituciones) ${ }^{4}$. Se puede hacer remontar esta fase teórico-sistemática del debate a 1969, es decir, a la fecha de IX Congreso Alemán de Filosofía, en el cual, en las exposiciones de Paul Lorenzen (Das Problem des Szientismus), de Jürgen Habermas (Bemerkungen zum Problem der Begründung von Werturteilen) y de Richard M. Hare (Wissenschaft und praktische Philosophie), la discusión de los problemas vinculados con el redescubrimiento de la filosofía práctica se desembarazó de la referencia a modelos del pasado, para comenzar a ser desarrollada a partir de puntos de vista, perspectivas y posiciones filosóficas más contemporáneas.

\section{LAS MOTIVACIONES Y LAS RAÍCES DEL NEO-ARISTOTELISMO}

Como quiera que sea respecto de este debate en su conjunto, se puede decir que su origen se sitúa esencialmente en posiciones filosóficas que, aunque bastante diferenciadas entre sí, resultaron pronto

2. Cf. N. Luhmann, Zweckbegriff und Systemrationalität (Mohr, Frankfurt am Main, 1968); cf. también la controversia con J. Habermas en Theorie der Gesellschaft oder Sozialtechnologie. Was leistet die Systemforschung? (Suhrkamp, Frankfurt am Main, 1971).

3. Cf. esp. K. Lonenz, Das Sogenannte Böse: Zur Naturgeschichte der Agression (G. Borotha-Schoeler, Wien, 1963).

4. Cf. A. Gehlen, Urmensch und Spätkultur (Athenäum, Bonn, 1956) y también Moral und Hypermoral (Athenäum, Frankfurt am Main-Bonn, 1969). 
englobadas bajo la designación unitaria de "neo-aristotelismo" Los dos textos más importantes a este respecto, los cuales puede considerarse que han sido el punto de partida del debate, son el bien conocido libro de Hannah Arendt: Vita activa, aparecido inicialmente en los Estados Unidos en 1958 con el título The Human Condition, y luego traducido al alemán en $1960^{6}$, y el igualmente célebre libro de Hans-Georg Gadamer: Wabrheit und Methode ${ }^{7}$, aparecido el mismo año. En Vita activa, H. Arendt llamaba la atención del pensamiento contemporáneo sobre la determinación aristotélica de la $\pi \varrho \hat{\alpha} \xi ı \varsigma$ y su carácter fundamental para la comprensión del fenómeno de lo político (distinguido de la política) contra la tiranía de la producción y del trabajo, que caracterizan el mundo moderno. También Gadamer, en un capítulo de Wabrbeit und Methode que posteriormente llegó a ser célebre ${ }^{8}$, subrayaba la actualidad de la ética de Aristóteles, en razón precisamente del interés que presenta la determinación aristotélica del saber que orienta el obrar y la vida

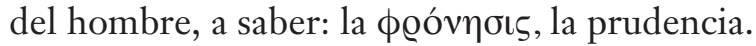

Estos textos no representan más que los dos ejemplos más famosos de una reasunción bastante generalizada de la comprensión

5. He examinado e intentado elucidar el particular sentido y el carácter problemático de esta designación en la comunicación: Che cosa significa neoaristotelismo?, presentada con ocasión de un seminario ítalo-alemán organizado en el mes de octubre de 1984 por la Universidad de Padua en Brixen (Südtirol), la cual se publicó primero con el título: La riabilitazione della filosofía pratica e il suo senso nella crisi della modernità, en un cuaderno de la revista "il Mulino" dedicado a los problemas de la ética contemporánea ("il Mulino" 35/6 (1986) 928- 949), y después nuevamente, con el título original, en E. BerTi (ed.), Tradizione e attualità della filosofía pratica (Marietti, Genova, 1988) 111-135. Cf. también la contextualización crítica propuesta por E. Berti, La philosophie pratique d'Aristote et sa "rehabilitation" récente, "Revue de Métaphysique et de Morale" 95 (1990) 249-266. Para un análisis de la asociación típicamente alemana de neoaristotelismo y conservadurismo, cf. H. Schnädelbach, Was ist Neoaristotelismus?, en el volumen colectivo W. Kuhlmann (ed.), Moralität und Sittlichkeit. Das Problem Hegels und die Diskursethik (Suhrkamp, Frankfurt am Main, 1986) 38-63. Schnädelbach, sin embargo, no cuestiona dicha asociación.

6. H. Arendt, The Human Condition (University of Chicago Press, Chicago, 1958); edición alemana: Vita activa Vom tätigen Leben (Kohlhammer, Stuttgart, 1960).

7. H.-G. Gadamer, Wabrheit und Methode. Grundzüge einer philosophischen Hermeneutik (Mohr, Tübingen, 1960).

8. Se trata del capítulo: Die hermeneutische Aktualität des Aristoteles (H.-G. Gadamer, op. cit., 295-307). 
aristotélica de la $\pi \varrho \hat{\alpha} \xi ı \varsigma$ y del saber ético y político que le concierne. Esta reasunción, fenómeno esencialmente alemán, ocurrió en un momento de crisis particularmente profunda tanto de las disciplinas que tradicionalmente se ocupaban del obrar humano, especialmente la ética y la política, como de las ciencias humanas y sociales, que, en nuestro siglo, se habían impuesto y afirmado cada vez más como el único saber referido al obrar del hombre que podía llamarse con razón científico.

A este respecto, el caso de la ciencia política puede considerarse paradigmático. En efecto, incluso tras haber obtenido una dignidad epistemológica propia, dicha ciencia se encuentra, sin embargo, en la época contemporánea sumergida en una crisis concerniente a sus fundamentos conceptuales y en un estado en el cual su identidad como disciplina resulta bastante endeble, y ello no solo por haberse vuelto cada vez más una ciencia puramente empírica y descriptiva, sino también en la medida en que queda desgarrada por una pluralidad conflictiva de métodos, que se proponen, todos al mismo tiempo, como los más eficaces y como los únicos capaces de proveer una descripción adecuada de los fenómenos de la política ${ }^{9}$. La obra de Hannah Arendt, del mismo modo que la de Leo Strauss y la de Eric Voegelin, representa a este respecto una atrevida denuncia de las aporías e ingenuidades de la ciencia política moderna, contra la cual estos autores reivindican la actualidad de la filosofía política clásica. Se podría decir otro tanto en lo que concierne a las ciencias humanas y sociales: aunque su nacimiento y su constitución como saber científico sean un acontecimiento bastante reciente, también ellas han sido atormentadas, desde su inicio, por problemas de método y de autorepresentación epistémica. Bastaría recordar en esta materia la polémica sobre el método (Methodenstreit) sociológico que se desarrolló durante los veinte últimos años del siglo pasado, polémica a la cual se encuentran ligados los nombres de Carl Menger y Gustav Schmoller, el debate sobre los juicios de valor (Wer-

9. Cf. R. H. Sснмidт (ed.), Methoden der Politologie (Wissenschaftliche Buchgesellschaft, Darmstadt, 1967); H. Schneider (ed.), Aufgabe und Selbstverständnis der politischen Wissenschaft (Wissenschaftliche Buchgesellschaft, Darmstadt, 1967). 
turteilstreit) que tuvo lugar a partir de 1909 en los Congresos del Verein für Sozialpolitik (Asociación para la Política Social) y de la Deutsche Gesellschaft für Soziologie (Sociedad Alemana de Sociología), que está ligado a la obra de Max Weber y Werner Sombart, la controversia sobre la sociología del saber, o también, por último, el debate sobre el método positivista o dialéctico en sociología, conocido bajo el nombre de Positivismusstreit, el cual vio oponerse a Adorno, Popper y sus respectivas escuelas ${ }^{10}$.

En relación con todos estos problemas, la reasunción de la filosofía práctica de la tradición aristotélica se propuso como una solución alternativa, en la medida en que fue reasumida en su valor paradigmático de modelo de saber alternativo a la modernidad y a la idea unitaria de ciencia que la caracteriza. En efecto, la tradición de la philosophia practica, que se puede hacer remontar a Aristóteles

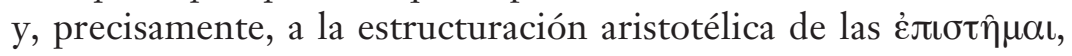
designa el campo del saber cuyo objeto es el obrar humano, campo delimitado tanto respecto de la philosophia theoretica como de la philosophia mechanica y de las artes. De acuerdo con la tradición que se consolida, sobre todo, en las enciclopedias medievales, dicho campo era tripartito: comprendía la ética, saber concerniente al obrar individual, la economía, saber concerniente al oîxos, es decir, la casa en su conjunto, y la política, saber concerniente al obrar en la comuni-

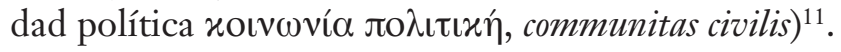

10. Cf. H. Albert, E. Topitsch (eds.), Werturteilsstreit (Wissenschaftliche Buchgesellschaft, Darmstadt, ${ }^{2}$ 1979); V. Meja, N. STehr (eds.), Der Streit um die Wissenssoziologie, 2 vols. (Suhrkamp, Frankfurt am Main, 1982); T. W. Adonno et al., Der Positivismusstreit in der deutschen Soziologie (Luchterhand, Neuwied-Berlin, 1969).

11. Esta tripartición del saber práctico no fue establecida por Aristóteles. Es cierto que

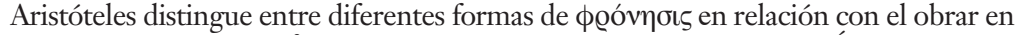
la đó $\lambda_{\iota}$, el obrar en el oîxos y el obrar del individuo (cf. por ejemplo Ética a Nicómaco [EN] VI 8). Pero, en general, si se quiere retener la idea de una articulación del saber práctico en Aristóteles, hay que pensar, más bien, en una bipartición de dicho saber en ética y política. La tripartición nació probablemente como consecuencia de la gran influencia del Oikonomikós pseudo-aristotélico. En cuanto a la clasificación general del saber en disciplinas teóricas, prácticas y poiéticas, cf. Tópicos VII 1 y Metafísica $\alpha 1$ (donde Aristóteles distingue entre disciplinas teóricas y prácticas); la tripartición completa se encuentra en Tópicos VI 6; VIII 1; Metafísica E 1; K 7; ENVI 2; la distinción entre ciencias prácticas y poiéticas está en De caelo III 7; Metafísica $\Lambda 9$. 
En realidad, independientemente de una referencia precisa y rigurosa a la clasificación aristotélica del saber, la tradición de la filosofía práctica como disciplina, con su tripartición, se constituyó y permaneció viva a partir de la Edad Media esencialmente en la cultura escolástica de las enciclopedias ${ }^{12}$ y en la estructuración en disciplinas del saber producido en las universidades alemanas ${ }^{13}$. En efecto, a partir de la mitad del siglo XIII con el nuevo descubrimiento de la Ética y de la Política de Aristóteles, la tendencia, hasta entonces dominante en la enseñanza universitaria, a tratar la moral y la política como de-

12. La tripartición del saber práctico en ética, economía y política - ligada a la clasificación aristotélica de las ciencias (teóricas, prácticas, poiéticas) y alternativa en relación con la articulación helenística del saber en lógica, ética y física (articulación que Cicerón atribuye a Platón, pero que Sexto Empírico hace remontar a Jenócrates) - es introducida en la cultura latina por Boecio (en su comentario a la Eisagogé de Porfirio) y se encuentra en casi todas las enciclopedias más importantes de la Edad Media, como, para nombrar solo las más conocidas, el De artibus ac disciplinis de CAsIodoro, las Etymologiae de Isidoro de Sevilla, el Speculum Quadruplex de Vincent DE Beauvais, el Didascalicon de Hugo de SAn Víctor, el De divisione philosophiae de Domenicus Gundissalinus, el De ortu scientiarum de Robert Kilwardby. Cf. J. MariéTan, Problème de la classification des sciences d'Aristote à St. Thomas (Felix Alcan, Paris, 1901); J. Kосн (ed.), Artes liberales. Von der antiken Bildung zur Wissenschaft des Mittelalters (Brill, Leiden, 1959, ${ }^{2}$ 1976); J. A. WeIsHeIPL, Classification of the Sciences in Medieval Thought "Mediaeval Studies" 27 (1965) 54-90; M. DE GANDILLAC (ed.), La pensée encyclopédique au Moyen Âge (Éditions de la Baconnière, Neuchátel, 1966); Arts libéraux et philosophie au Moyen Age, Actes du IVe Congrés international de Philosophie médiévale, Université de Montréal, Canada, 27 août-2 septembre 1967 (Institut d'Études Médiévales-Librairie Philosophie J. Vrin, Montréal-Paris, 1969); I. НAdot, Arts libéraux et philosophie dans la pensée antique (Études Augustiniennes, Paris, 1984). La tripartición del saber práctico según la clasificación aristotélica de las ciencias es retomada también por la tradición enciclopédica moderna desde F. Bacon, pero en este caso aparece, además, combinada con la tripartición del saber, de procedencia helenística, en lógica, física y ética. Cf. U. DiERse, Enzyklopädie: Zur Geschichte eines philosophischen und wissenschaftlichen Begriffs (Bouvier, Bonn, 1977); W. Schmidt-Biggemann, Topica universalis. Eine Modellgeschichte bumanistischer und barocker Wissenschaft (Meiner, Hamburg, 1983).

13. Cf. los trabajos de H. MaIER, Die Lebre der Politik an den älteren deutschen Universitäten, en D. ОвеRndörfer (ed.) Wissenschaftliche Politik. Eine Einfübrung in Grundfragen ibrer Tradition und Theorie (Rombach, Freiburg, 1962) 59-116 y en H. MaIER, Politische Wissenschaft in Deutschland: Lebre und Wirkung (Piper, München, 1985) 31-67; H. MaIER, Die ältere deutsche Staats- und Verwaltungslehre (Polizeiwissenschaft) (Luchterhand, Neuwied-Berlin, 1966 / Deutscher Taschenbuch, Munchën, 1986); de modo más general véase el clásico estudio de H. Demifle, Die Entstehung der Universitäten des Mittelalters bis 1400 (Weidmann, Berlín, 1885, reprod. facs. Akademische Druck- u. Verlagsanstalt, Graz, 1956), y la rica bibliografía contenida en R. Graf von Westphalen, Akademisches Privileg und demokratischer Staat. Ein Beitrag zur Geschichte und bildungspolitischen Problematik des Laufbahnwesens in Deutschland (Klett-Cotta, Stuttgart, 1979). 
pendientes de la metafísica y de la teología sufrió una inversión; pudieron reunirse entonces las condiciones previas indispensables para la constitución de una autonomía de la philosophia practica, al menos, en cuanto disciplina ${ }^{14}$. En las universidades, efectivamente, junto a las lecciones del organicus, en las cuales se estudiaba los libros del Organon, y las del philosophus naturalis y del metaphysicus, el estudio de la filosofía comprendía también las lecciones del ethicus, es decir, el estudio de la Ética y la Política; más tarde se agregó también el estudio de la economía y la crematística. La philosopbia practica se transformó así en una materia oficial de enseñanza y, al menos en Alemania, siguió siéndolo casi hasta el fin del siglo XVIII ${ }^{15}$.

14. Desde 1215, la ética era enseñada en París como una disciplina autónoma, en la facultad de artes. En un comienzo, la enseñanza se apoyaba en la traducción de los libros II y III 1 de la EN, es decir, en la ethica vetus, que comprendía, sobre todo, la teoría de las virtudes; luego, se utilizó también el libro I, la etbica nova, que comprendía la doctrina de la felicidad; por último, se tuvo a disposición la traducción completa de Roberto Grosseteste (1246/1247). Después de la traducción de la ethica nova, el esfuerzo por conciliar la ética aristotélica con la teología fue una tarea particularmente dura, que condujo a una reducción de la doctrina aristotélica de la $\varepsilon v ̀ \delta \alpha \iota \mu v i ́ \alpha$ y a su subordinación a la doctrina teológica de la beatitudo celestial; esto implicó también una comprensión teórica de la ética en el sentido de la etbica docens más bien que en el de la ethica utens. Sobre estos problemas, tras los estudios de pioneros como M. Grabmann, Ph. Delhaye y O. Lottin, se dispone ahora de la excelente monografía de G. Wieland, Ethica, Scientia practica: Die Anfänge der philosophischen Ethik im 13. Jahrbundert (Aschendorff, Münster, 1981). Los estudios de $\mathrm{Ph}$. Delhaye fueron reunidos recientemente bajo el título: Enseignement et morale au XIIe siécle (Éditions universitaires-Éditions du Cerf, Fribourg-Paris, 1988).

15. Las razones que motivan esta persistencia son numerosas y bastante complejas: la influencia del proceso de aristotelización del pensamiento reformista por parte del praeceptor Germaniae $\mathrm{Ph}$. Melanchton; la resistencia que la cultura política alemana opuso a la penetración de la idea moderna del Estado de tipo maquiavélico o hobbesiano, la cual representaba la antítesis más poderosa frente a la concepción aristotélica de la política como ciencia práctica; finalmente, la asimilación progresiva de elementos innovadores como la tradición iusnaturalista o la cameralística y la nueva economía, que fueron retomadas en Alemania en el seno de la tradición de la philosophia practica. Sobre la difusión del aristotelismo político en Alemania, además de los estudios de H. Maier citados, véase: M. Riedel, Metaphysik und Metapolitik. Studien zu Aristóteles und zur politischen Sprache der neuzeitlichen Philosophie (Suhrkamp, Frankfurt am Main, 1975); V. Sellin, Politik en O. Brunner, W. Conze, R. Koselleck (eds.), Geschichtliche Grundbegriffe: Historisches Lexikon zur politisch-sozialen Sprache in Deutschland, vol. 4 (Klett-Cotta, Stuttgart, 1978) 789-874; por último, en general, P. Petersen, Geschichte der aristotelischen Philosophie im protestantischen Deutschland (Meiner, Leipzig, 1921). 
En esta tradición de enseñanza escolástica y universitaria de la philosophia practica, que se inspiraba en Aristóteles, pero que se alejaba crecientemente del aristotelismo, el tratado de Christian Wolff: Philosophia practica universalis (1738-1739) ocupa un lugar especial y puede ser considerado como el último gran testimonio de dicha tradición: la philosophia practica es llamada aquí universalis ${ }^{16}$, pues provee el fundamento de la tripartición formal de las disciplinas prácticas, en cuanto está en la base de la Philosophia moralis sive Ethica, de la Económica y de la Philosophia civilis sive Politica. El tratado de Wolff representa, en efecto, el último esfuerzo sistemático por estructurar y organizar las disciplinas prácticas según el modelo aristotélico del saber, aunque de aristotélico en sentido propio no resta aquí más que el andamiaje formal exterior y la distribución de la materia, y ello a pesar del hecho de que el método profesado por Wolff es el matemático ${ }^{17}$.

\section{La alternativa moderna a la Filosofía práctica}

Por otra parte, esta "tradición" es una tradición perdedora frente a la opción predominante de la modernidad. En efecto, desde una perspectiva muy general, que deja de lado una consideración más precisa de las complejas transformaciones que la caracterizan, puede decirse que en la configuración del sistema moderno del saber según

16. En la Ratio praelectionum Wolffianarum in mathesin et Philosophiam universam (Renger, Halae Magdeburg, 1718), Wolff observa a este respecto: "Philosophiae practicae universalis nomen hactenus inauditum inter Philosophos, nec minus res ipsa ignorata: mihi tamen haec disciplina utilis ac necesaria videtur ad Philosophiam practicam rite constituendam" (VI, 2).

17. Más allá de la tradición escolástica y universitaria, de la cual Wolff puede ser considerado como el último gran representante, habría que examinar la presencia del aristotelismo en otros ámbitos de la transmisión del saber, que se expresan, por ejemplo, en géneros literarios como la literatura de los "espejos del príncipe", la Hausväterliteratur la literatura del buen comportamiento y de la "conversación ciudadana”. Sobre este punto, véase las investigaciones de E. R. Curtius, Europäische Literatur und lateinisches Mittelalter (Francke, Bern-München, 1948), para lo que concierne a la literatura; los trabajos de O. Brunner, Neue Wege der Verfassungs- und Sozialgeschichte (Vandenhoeck \& Ruprech, Gottingen, ${ }^{2} 1968$ ), y Adeliges Landleben und europäischer Geist (Otto Mueller Verlag, Salzburg, 1949), siguen siendo fundamentales en lo que concierne a la historia. 
el ideal de la scientia se produce un cambio radical sea de la autocomprensión, sea del valor posicional en la clasificación general del saber, en la disciplinas concernientes al obrar humano. Cambia su lugar por relación al que esas disciplinas tenían en el sistema an-

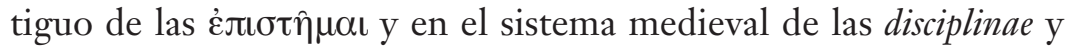
las artes. Entre los diversos factores que caracterizan este cambio debe atribuirse un valor paradigmático a la identificación de scientia y theoria y a la afirmación de la idea de método como garantía de universalidad y de rigor científico. Se puede decir que la aplicación del método a la ciencia concebida como teoría, es decir, la asunción del método como parámetro ideal del saber en el cuadro de una comprensión teorética del saber mismo tuvo un impacto destructor sobre la estructuración del dominio del saber que, tradicionalmente, había sido designado como philosophia practica y distinguido, en tanto tal, de la philosophia theoretica y de la philosophia mechanica o de las artes.

Ahora bien, fundándose en la presuposición de que el método matemático representa el paradigma del saber en general, hay dos consecuencias posibles: por una parte, la constatación de la inaplicabilidad del método al dominio de la philosophia practica, dicho de otro modo, al campo del saber que orienta el obrar, lleva a concebir a esta como una suerte de philosophia minor, como un saber que tiene un carácter tan solo provisorio y aproximativo; por otra parte - y tal es la opinión predominante en la modernidad- el obrar humano es tomado como objeto de saber no en el sentido de que dicho obrar sea orientado de modo concreto por tal saber, sino en el sentido de que se lo toma como el campo de un posible ejercicio del conocimiento, dicho de otro modo, se toma al obrar humano como un mundo que, al igual que el de la naturaleza, puede ser captado y descripto rigurosamente en sus mecanismos y en su funcionamiento. Se tiene entonces la idea de una aplicación de un saber tan riguroso como el saber matemático a la ética (Spinoza), a la política (Hobbes), al derecho (Pufendorf) y a la economía (Petty).

El pensamiento kantiano, punto crucial en el desarrollo de la filosofía moderna, representa, en adhesión a la idea moderna de la razón, la tentativa más consistente de corregir esta tendencia. 
Puesto que en el marco de este artículo es imposible examinar cómo Kant propone una comprensión del saber práctico que difiere tanto de la tradición objetivista paleo-moderna como de la de la philosophia practica de procedencia aristotélica, baste con recordar la claridad y la decisión con las que Kant separa la consideración del hombre como ser perteneciente al orden de la naturaleza, y sometido entonces a la causalidad física, de la consideración del hombre como perteneciente al orden de la libertad. De aquí procede la distinción kantiana entre el uso teórico y el uso práctico de la razón, entre la metafísica de la naturaleza y la metafísica de las costumbres, entre la consideración fisiológica y la consideración pragmática del hombre: una apunta a determinar "lo que la naturaleza hace del hombre", la otra "lo que el hombre como ser libre hace o bien puede y debe hacer de sí mismo" ${ }^{18}$. Lo que se debe subrayar aquí es que, a través de su consideración práctico-moral del obrar, Kant pretende restituir a este saber un carácter de orientación, de suerte que introduce así un cambio notable respecto de la tendencia predominante en la época moderna, según la cual se considera el obrar humano como objeto posible de un saber científico riguroso, el cual es únicamente constatativo y descriptivo.

Por referencia a este desarrollo, la atribución de un carácter científico a las Geisteswissenschaften — término que nace como traducción del inglés moral sciences ${ }^{19}$ - no representa una ruptura, sino más bien una continuación y una radicalización. En efecto, la extensión del ideal de un saber riguroso y universal a toda realidad exige, para ser eficaz, que se reconozca la diversidad y la especificidad de los dominios científicos y, por consiguiente, que se renuncie a la unidad del método: es justamente esta tematización de los caracteres específicos de las ciencias del espíritu lo que Dilthey define

18. "Die physiologische Menschenkenntnis geht auf die Erforschung dessen, was die Natur aus dem Menschen macht, die pragmatische auf das, was er ais freihandelndes Wesen aus sich selbst macht, oder machen kann und soll"; I. KaNT, Anthropologie in pragmatischer Hinsicht, en Werke: Akademie-Textausgabe vol. 7 (Reimer, Berlin, 1917) 117.

19 Cf. K. C. Köнnke, Entstehung und Aufstieg des Neukantianismus: Die deutsche Universitätsphilosophie zwischen Idealismus und Positivismus (Suhrkamp, Frankfurt, 1986) 468, n. 64. 
como la tarea de una "crítica de la razón histórica", que permita obtener un saber científico, es decir, objetivo y riguroso, en el dominio de las res gestae, pues una aplicación del método científico aparecía, en este caso, como impracticable o como reductiva. Pero lo que no cambia, sino que, por el contrario, resulta aquí radicalizado, es la manera típicamente moderna de considerar el obrar humano y la relación entre el obrar y el saber que lo considera. En efecto, el obrar sigue siendo aquí objeto de una consideración descriptiva, dicho de otro modo, es objetivado y reificado como campo de un análisis constatativo y veritativo; a su vez, el saber correspondiente no es ya un saber práctico-moral capaz de orientar el obrar, sino que se convierte simplemente en observación y descripción teórica y neutra de las dinámicas y las regularidades que lo caracterizan. No orienta el obrar indicándole sus fines, sino que ve y prevé las acciones, tal como el físico ve y prevé los movimientos de la naturaleza.

Dicho de otro modo, es precisamente para garantizar la eficacia de la asociación entre verdad y método para lo que hace falta diversificar la metodología de análisis científico por referencia a los objetos específicos considerados; pero en esta diferenciación el ideal de saber presupuesto por las ciencias de la naturaleza y por las ciencias del espíritu (de la cultura, del hombre), por las disciplinas nomotéticas y por las disciplinas idiográficas permanece idéntico. Es el ideal de un saber riguroso, objetivo, universal, neutro, guiado y controlado por el método. Se sigue, como ha subrayado Gadamer, que incluso las experiencias extra-metódicas de la verdad como el arte, la historia, la moral, la política son re(con)ducidas al horizonte de un saber metódico y científico. El saber que orienta la moral, la ética - las moral sciences - se convierte entonces en el saber neutro de las ciencias del espíritu, de las ciencias de la cultura o del hombre. Su consideración del obrar se conforma al ideal de la objetividad y la universalidad descriptiva del saber científico; la consideración práctico-moral de otros tiempos se transforma en una consideración teórico-constatativa y pierde así el contenido de verdad que le había pertenecido en la tradición anterior.

Esta transformación es definitivamente sancionada por la célebre tesis de la neutralidad axiológica (Wertfreibeit). La defini- 
ción del carácter descriptivamente neutro del saber de las ciencias humanas y sociales, definición estrechamente ligada al reconocimiento de su carácter científico, despoja a este saber de toda función práctico-orientativa, dicho de otro modo, lo despoja de la conexión con aquellos valores que —según una idea también típicamente moderna ${ }^{20}$ - deberían orientar el obrar, las elecciones y la vida humana en su conjunto. La neutralidad descriptiva se convierte en un cierto momento en un carácter inmanente a la razón en tanto tal, que, de este modo, resulta progresivamente desposeída de sus contenidos sustanciales y se desarrolla así en dirección de la mera instrumentalidad y la mera funcionalidad. Al volverse incapaz de orientarla, la razón pierde su importancia para la vida, su Lebensbedeutsamkeit.

\section{LA OPOSICIÓN DEL NEO-ARISTOTELISMO A LA MODERNIDAD}

Contra este desarrollo y contra esta comprensión moderna del obrar los neo-aristotélicos alemanes han proclamado la necesidad de rehabilitar la filosofía práctica de la "tradición aristotélica", a pesar de todas las dificultades que hay para reconocer una connotación esencial y continua que caracterice dicha tradición en un sentido efectivamente aristotélico. En realidad, sin manifestar una conciencia explícita y rigurosa de la determinación aristotélica del saber práctico, los neo-aristotélicos han abrevado libremente en ella, siguiendo exigencias y perspectivas bastante diferentes, para extraer elementos aptos para diseñar una comprensión de la racionalidad práctica capaz de oponerse a y, en definitiva, de corregir la idea moderna de un saber unitario y metódico, objetivo y descriptivo, aplicable al ser en su conjunto. Por ello, la reasunción de la

20. Como lo ha mostrado, sobre todo, Heidegger: cf. en M. Heidegger, Einfuibrung in die Metaphysik (1935), en Gesamtausgabe vol. 40, (Klostermann, Frankfurt am Main, 1983), \$ 57, 205-208; Nietzsches Wort “Gott ist tot” (1943), en Gesamtausgabe vol. 5 (= Holzwege), 209-267, 227 s.; cf. también Brief über den Humanismus, en Gesamtausgabe vol. 9 (=Wegmarken), 313-364, 349s.; por último, véase también C. Sснмітт, Die Tyrannei der Werte, en Säkularisation und Utopie: Ebracher Studien, E. Forsthoff zum 65. Geburtstag (Kohlhammer, Stuttgart, 1967) 37-62. 
concepción aristotélica de la racionalidad práctica ha sido determinada por el punto de vista que la motivaba y por la expectativa general en la que se encontraba situada, es decir, por la idea de poder utilizar esta concepción como respuesta a la crisis de la comprensión moderna del saber. Por lo demás, la atención dirigida a la filosofía práctica aristotélica adquiere diferentes inflexiones, según los diferentes campos disciplinarios en los que se efectúa. Hay que decir, pues, que la designación de conjunto "neo-aristotelismo" debe ser tomada en términos bastante generales, tanto más cuando los neo-aristotelismos se limitan a retomar solo ciertos aspectos o determinaciones particulares, aislados del contexto de la comprensión aristotélica del saber y utilizados en función de las cuestiones que los ocupan.

Un panorama de las posiciones que han contribuido a despertar el interés por la filosofía práctica de Aristóteles y que, por ello, pasan de algún modo, sobre todo en Alemania, por neo-aristotelismos indica, mejor que cualquier análisis, el carácter relativo de esta connotación colectiva: la actualidad de la filosofía práctica aristotélica ha sido redescubierta en el dominio de las ciencias políticas (Leo Strauss, Eric Voegelin, Hannah Arendt, Wilhelm Hennis, Hans Maier); de igual modo, en el dominio de las ciencias históricas, la referencia a los antiguos órdenes sociales en los que la filosofía práctica había sedimentado motivó investigaciones de historia social que tematizaron - por oposición a la visión progresista de la historia- la transición del mundo vetero-europeo medieval al mundo moderno de la sociedad burguesa industrial (Otto Brunner, Werner Conze); en las ciencias del derecho, la atención dedicada a Aristóteles llevo a retomar el método tópico-dialéctico (Theodor Viehweg, Chaïm Perelman); en las ciencias pedagógicas, motivó una rehabilitación de la tradición humanista, retórica y literaria (tradición para la cual fue importante el debate que tuvo lugar a comienzos de los años sesenta del siglo XX entre Joachim Ritter y Helmut Schelsky en torno a la función de las Geisteswissenschaften en la Universidad); más recientemente aún, merecen ser señalados los estudios de Jürgen-Eckhart Pleines, alumno de Gadamer; por último, la filosofía práctica de Aristóteles concentró de modo 
muy notable la atención también en el ámbito de la filosofía misma (Hans-Georg Gadamer, Joachim Ritter, Günther Bien, Klaus Held, Rüdiger Bübner).

\section{LAS TESIS PROGRAMÁTICAS FUNDAMENTALES DEL NEO-ARISTOTELISMO}

Frente a esta situación de interés generalizado y en lugar de demorarse en el examen de posiciones diversas y variadas, conviene realizar un intento por reconocer cuáles son los elementos que caracterizan y reúnen estas posiciones, de modo que permiten calificarlas de "neo-aristotelismo". Me parece que dichos elementos característicos pueden ser reconducidos a tres intuiciones epistémicas o a tres tesis fundamentales que los neo-aristotélicos retoman a partir

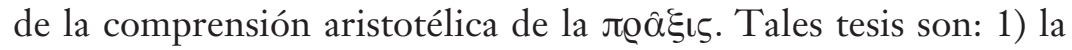
afirmación de la autonomía de la $\pi \varrho \hat{\alpha} \xi ı \varsigma$ respecto de la $\theta \varepsilon \omega \varrho i ́ \alpha ; 2)$

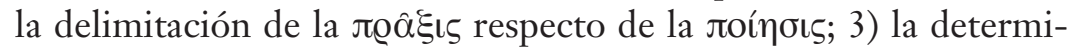
nación de las características específicas del saber de la $\pi \varrho \hat{\alpha} \xi \iota \varsigma$, de la racionalidad práctica.

\section{a) $\Theta \varepsilon \omega \varrho i \alpha$ y $\pi \varrho \hat{\alpha} \xi \iota \varsigma$}

Se sabe que Aristóteles, en oposición a Platón, marca por primera vez de modo sistemático la diferenciación entre la actitud del

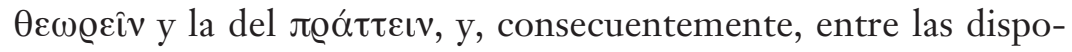
siciones científicas correspondientes propias del saber práctico y del saber teorético. Esta diferenciación se efectúa con la ayuda de criterios, sin duda alguna, estrechamente ligados, desde el punto de vista histórico, a la clasificación y la comprensión aristotélica del saber, pero poseedores en sí mismos de una plausibilidad específica propia, a la cual se remiten los neo-aristotélicos. Tales criterios son:

a) La diversidad del fin perseguido en cada caso: en la $\theta \varepsilon \omega \varrho i ́ \alpha$ tal fin es la verdad ( $\dot{\alpha} \lambda \hat{\eta} \theta \varepsilon \iota \alpha)$, en la $\pi \varrho \hat{\alpha} \xi ı \varsigma$ es el éxito de la $\pi \varrho \hat{\alpha} \xi ı \varsigma$

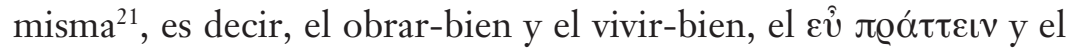


$\varepsilon \hat{v} \zeta \hat{\eta} v$. Esto implica una diferencia en el estatuto epistemológico de las disciplinas científicas que corresponden a estas dos actitudes: las ciencias teoréticas efectúan una consideración contemplativa y constatativa de la verdad de las realidades naturales y celestes que no dependen del hombre, mientras que el saber práctico — que tiene igualmente el carácter de saber científico- está orientado hacia la comprensión de la $\pi \varrho \hat{\alpha} \xi ı \zeta$, de la cual persigue el éxito ${ }^{22}$.

b) Las diferentes modalidades de ser del ente respectivamente considerado: en el caso de las ciencias teóricas, el ente considerado posee una estabilidad de ser superior a la del obrar humano, que es objeto de la filosofía práctica; las acciones humanas, en efecto, no

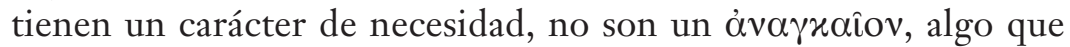
no puede ser de otro modo que como es ( $\tau \grave{\alpha} \mu \grave{\eta} \dot{\varepsilon} v \delta \varepsilon \chi o ́ \mu \varepsilon v \alpha$ ő $\lambda \lambda \omega \varsigma$

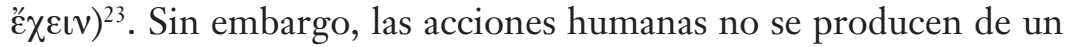

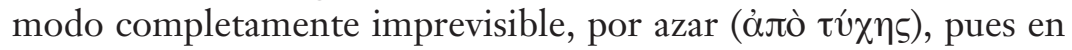
tal caso, según el principio aristotélico de que no hay ciencia del accidente, no sería posible someterlas a una consideración epistémica; el carácter de ser de las acciones humanas, objeto de la ciencia práctica, se sitúa, pues, entre lo necesario y lo accidental, en el dominio que posee la regularidad relativa de lo que, como dice Aristóteles,

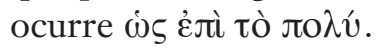

c) Los diferentes grados de precisión ( $\left.\dot{x} \varrho i^{\prime} \beta \varepsilon \iota \alpha\right)$ que se puede obtener $^{24}$ : por referencia a la constitución móvil y variable de su propio objeto, las ciencias prácticas no pueden alcanzar el mismo grado de precisión que es posible, por ejemplo, en las matemáticas; pero, puesto que esta menor precisión no depende de un defecto del conocimiento, sino más bien de la naturaleza misma del ente que es objeto de dicho conocimiento, Aristóteles no niega la cientificidad del saber práctico; no la considera, como se hará luego en la tradición escolástica, como una especie de cientificidad menos fuerte, como si la philosophia practica fuera una suerte de philosophia minor: el saber

22. EN VI 2, 1139a26-27.

23. Cf. por ejemplo, ENVI 3, 1140a1-2.

24. Cf. sobre todo EN I 1, y II 2; IX 2, 1165a12-14; véase también H. Bonitz, Index aristotelicus (Akademische Druck- u. Verlagsanstalt, Graz, ${ }^{2} 1955$ ) 27b-28a (sub voce

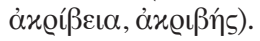


práctico es, por cierto, un saber solamente verosímil, pero no en el sentido de que fuera una aproximación a lo verdadero (verisimilitudo) que no alcanza nunca la verdad, sino más bien en el sentido de que es exactamente la verdad propia de lo probable (probabilitas).

d) La diferencia de las modalidades de argumentación respectivamente practicadas: en razón de los caracteres específicos que lo determinan, el saber práctico no se estructura de un modo apodíctico, sino más bien de un modo tópico-dialéctico; si se toma por ejemplo el silogismo práctico, se puede decir que este tiene el

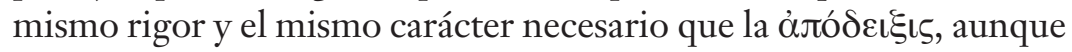
no se funda en premisas necesarias y verdaderas, sino solo en premisas probables, en opiniones notables o generalmente aceptadas ( $\varepsilon^{\prime} v \delta \circ \xi \alpha$ ), de suerte que las conclusiones a las cuales llega no pueden tener ellas mismas sino un carácter probable, tópico-dialéctico. Aunque, desde una perspectiva general, este punto parece plausible, no ignoro que es bastante controvertido, ni olvido que para dar una respuesta satisfactoria sobre esta cuestión sería necesario distinguir entre la racionalidad de la prudencia que orienta el obrar y la racionalidad del conocimiento científico del obrar ${ }^{25}$.

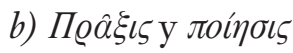

En Aristóteles, y una vez más en oposición a Platón ${ }^{26}$, se encuentra la

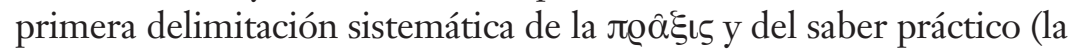

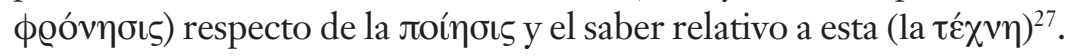

25. Para esta cuestión y, en general, para un estudio de la comprensión aristotélica

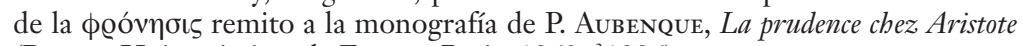
(Presses Universitaires de France, Paris, 1963, ${ }^{3} 1986$ ).

26. Platón clasifica el saber científico, según una dicotomía, en ciencias teoréticas y ciencias prácticas, pero su caracterización de lo "práctico" es radicalmente dife-

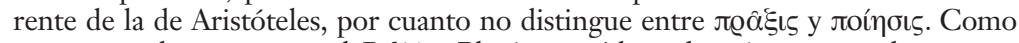
se ve muy claramente en el Político, Platón considera el carácter manual como un criterio para distinguir el saber práctico del saber teorético. El saber práctico es, pues, esencialmente el saber de las técnicas; las ciencias teoréticas son, a su vez, de dos géneros: o bien dan lugar a juicios, o bien producen realidades nuevas que no existían de antemano: la política es considerada como una ciencia teorética de este tipo, y es teorética porque no implica el uso de las manos (Político, 259e8-11).

27. Cf. EN VI 4-5. 
La dificultad y, al mismo tiempo, la importancia de esta delimitación

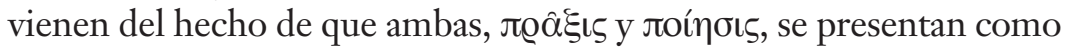
un obrar orientado hacia un fin; del mismo modo, los dos tipos de co-

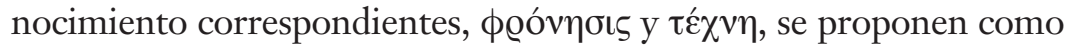
un saber capaz de orientar el obrar hacia el éxito. La diferencia se puede reconocer en la constatación del éxito del obrar mismo: en el caso de la лoí $\sigma ı \varsigma$, el éxito puede ser reconocido en el producto, en el ع̋orov, que permanece ahí después de que la acción poiética ha sido

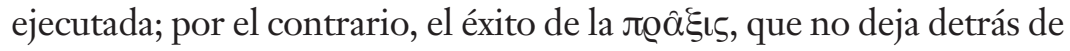
sí ningún producto concreto constatable, puede ser mensurado solo

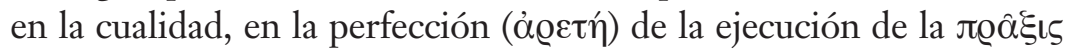
misma (que es una xívๆoıs y, en su forma perfectamente lograda,

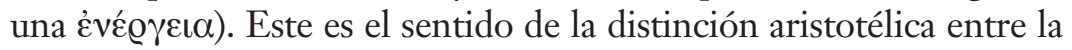
лoí $\sigma ı \varsigma$, concebida como un hacer que no tiene su fin en sí mismo

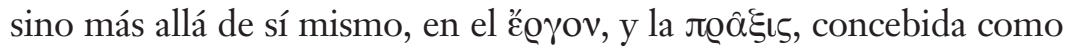
un hacer que tiene su fin en sí mismo, es decir, en la perfección de su ejecución. En consecuencia, hay un lazo estrecho entre el ser moral

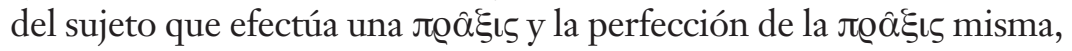
mientras que la perfección del ع̋@o

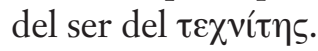

\section{c) La especificidad del saber de la $\pi \varrho \hat{\alpha} \xi_{l \zeta}$}

La especificidad del saber práctico exige una distinción tanto respecto del saber teorético como respecto del saber técnico. La primera demarcación, tal como acabamos de examinarla, da cuenta muy claramente de la distinción de la $\pi \varrho \hat{\alpha} \xi ı \varsigma$ y de la $\theta \varepsilon \omega \varrho i ́ \alpha$ por referencia al ente, tal como ellas, respectivamente, lo conocen. La segunda demarcación no puede fundarse en la diferente naturaleza ontológica del ente considerado, pues tanto la $\tau \dot{\varepsilon} \chi \nu \eta$ como

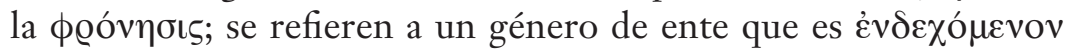

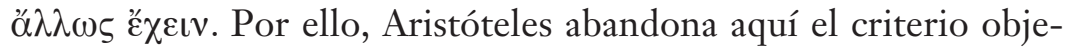

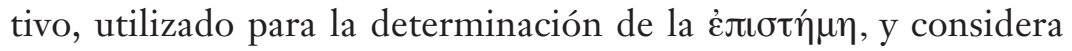
la diversidad de la disposición subjetiva sobre la cual se fundan

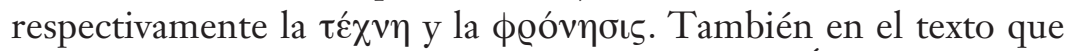
resulta capital para este punto, el libro VI de la Ética a Nicómaco, 
Aristóteles separa terminológica y conceptualmente, de un modo muy riguroso, el saber que se refiere a las acciones humanas, a las

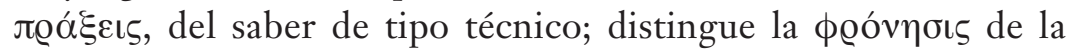
$\tau \hat{\varepsilon} \chi \vee \eta$. Esto no deja de ser un problema, pues, incluso en este texto, Aristóteles parece fundar esta división no de modo directo, sino remitiendo al hecho de que estas dos especies de saber se refieren a dos especies diferentes de hacer, ya distinguidas la una de la otra. Con todo, Aristóteles proporciona aquí elementos indicativos para una distinción, si no sistemática, al menos, digamos, fenomeno-

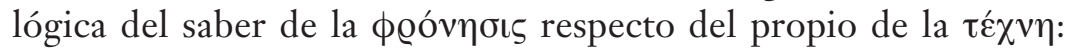

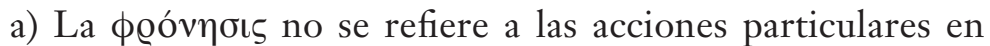
tanto tales, sino en tanto contribuyen al logro de la vida feliz en su

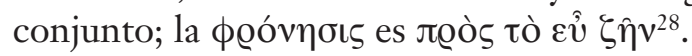

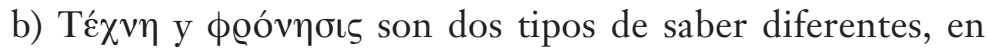
tanto se refieren a los dos géneros diferentes de la Joínoıs y de la

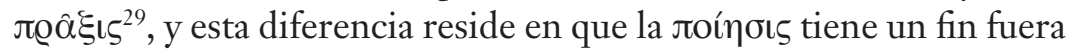
de ella misma, mientras que la $\pi \varrho \hat{\alpha} \xi ı \varsigma$ tiene su fin en su propio éxito $(\varepsilon \dot{u} \pi \varrho \alpha \xi i ́ \alpha)^{30}$.

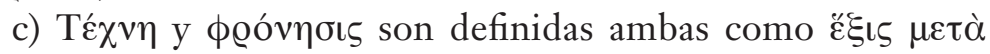

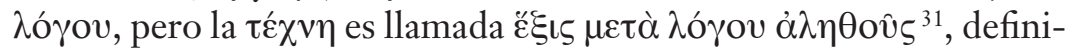
ción en la cual la verdad es elemento constitutivo del $\lambda$ ó $\gamma$ os, que va

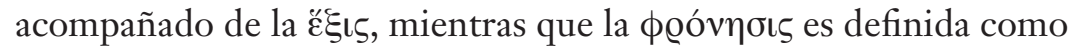

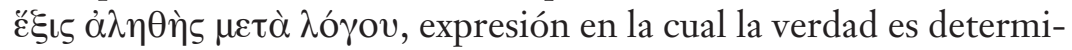
nación de la «̌̋ı misma $^{32}$. De este modo, Aristóteles probablemente quiere subrayar la importancia para el saber práctico-moral de la

28. EN VI 5, 1140a28.

29. EN VI 5, 1140b3-4.

30. EN VI 5, 1140b6-7.

31. ENVI 5, 1140a20-21.

32. EN VI 5, 1140b5, repetido en la línea $1140 \mathrm{~b} 20$ exactamente con la misma atribución de $\dot{\alpha} \lambda \eta \theta \hat{\eta}$ a la $\ddot{\varepsilon} \xi ı$. No hay razón, en mi opinión, para no conservar esta diferencia de

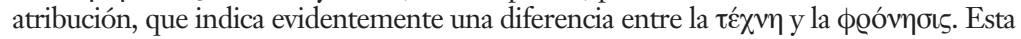
diferencia queda completamente suprimida si se acepta la sugerencia de Susemihl y de Stewart (seguida por Apel y Tricot y rechazada por Gauthier-Jolif), quienes corrigen dos veces $\dot{\alpha} \lambda \eta \theta \hat{\eta}$, en concordancia con $\varepsilon \xi \xi ı$, sustituyéndolo por $\dot{\alpha} \lambda \eta \theta$ ov̂ a fin de ponerlo en concordancia con $\lambda$ ó $\gamma o v$. Esto establece una analogía demasiado estrecha con la definición de la $\tau \varepsilon ́ \chi v \eta$, analogía que hace correr el riesgo de suprimir la diferencia que Aristóteles expresa aquí por medio de la sutileza terminológica de su definición. 


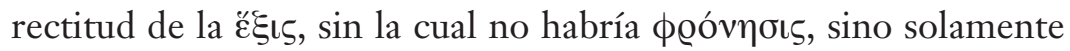

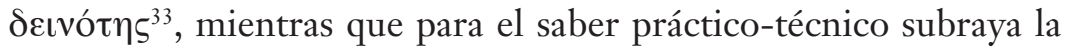

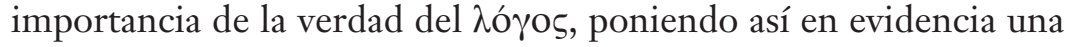
diferencia en la analogía de la definición de ambos tipos de saber. Por lo demás, sería necesario, en estrecha conexión con lo anterior, tomar en consideración el hecho de que la $\tau \dot{\chi} \chi v \eta$ puede ser efectuada en los contrarios (por ejemplo: el médico conoce los medios tanto para curar como para provocar la muerte), mientras que la ф@óvๆoıs no puede ser tal más que en la realización virtuosa de la acción; del mismo modo, está el hecho de que, como subraya Aristóteles, en la $\tau \dot{\varepsilon} \chi v \eta$ es preferible el que se equivoca voluntariamente,

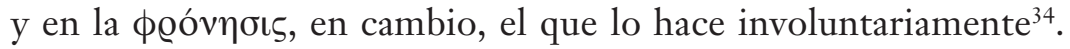

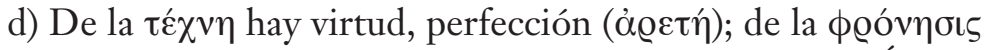
$\mathrm{no}^{35}$. Esta afirmación puede añadirse a la tesis, expuesta en Ética a

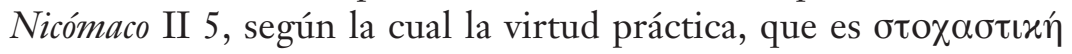

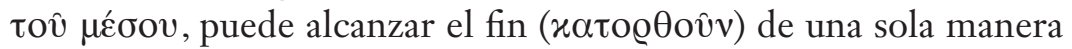
( $\mu \nu \alpha \chi \hat{\omega} \varsigma)$, mientras que es posible equivocarse de muchas maneras $(\pi \circ \lambda \lambda \alpha \chi \hat{\omega} \varsigma)^{36}$; del mismo modo, no puede haber diferentes grados

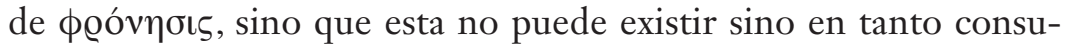

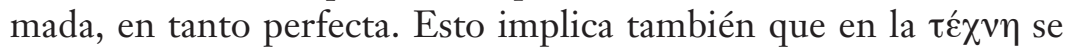
alcanza la perfección ejercitándose incluso a través de los errores; en la ф@óvๆoıৎ no se aprende a través de los errores, dicho de otro modo, no se llega a ser virtuoso practicando los vicios.

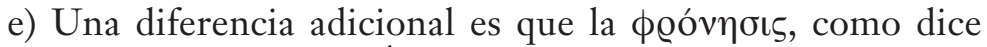
Aristóteles hacia el final de Ética a Nicómaco VI 5, no es solamente

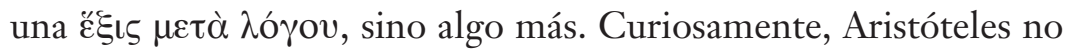
dice en qué consiste este "algo más", sino que se limita a dar una aplicación, diciendo que, si bien toda $\check{\varepsilon} \xi ı$ s puede ser olvidada, la

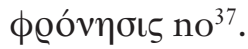

33. Cf. EN VI 13, 1144a23-29.

34. EN VI 5, 1140b22-24.

35. EN VI 5, 1140b24-25.

36. EN II 5, 1106b26-31.

37. EN VI 5, 1140b28-30. 
Sobre la base de estas indicaciones, es cierto que, aun cuando en los tratados de ética emplea muy a menudo ejemplos extraídos de las técnicas, sobre todo de la medicina, Aristóteles no asimila, sin

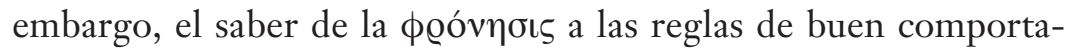
miento, a un saber técnico, como si el saber moral consistiera en la aplicación de un modelo, de un عỉoos, del hombre perfecto a las situaciones particulares de la vida humana ${ }^{38}$. Del mismo modo, la política, en tanto saber práctico, no puede ser reducida a una técnica de la conservación de la vida (o del poder), sino que encuentra su lugar propio en el espacio que se abre, una vez que está satisfecho y cumplimentado el sistema de las necesidades de la vida (oîxovouí $\alpha$ ), sobre el problema de la elección de la mejor forma posible de vida, sobre el problema del bien vivir $\varepsilon \hat{v} \zeta \hat{\eta} v$. El problema que, en mi opinión, queda abierto es el de la distinción entre la ф@óvๆoıs y la ciencia práctica, distinción que Aristóteles mismo subraya ${ }^{39}$, pero que, en general, en los neo-aristotélicos alemanes, tiende a ser abolida. Solo puede comprenderse de modo satisfactorio esta distinción si se precisa suficientemente la particularidad de la deter-

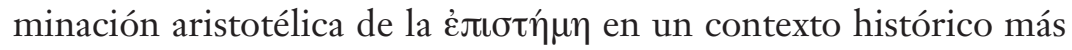
general, vale decir, sea por referencia a la comprensión platónica

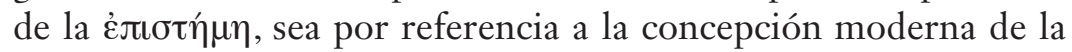
scientia, en tanto identificada con la $\theta \varepsilon \omega \varrho i ́ \alpha$.

38. Sobre este punto Gadamer ha dado, en el citado capítulo de Wabrheit und Methode, indicaciones fundamentales, que evidentemente ha derivado de la interpretación del libro VI de la Ética a Nicómaco ofrecida por el joven Heidegger en sus cursos (en particular, en la parte introductoria del curso de Marburgo del semestre de verano de 1924), que Gadamer conocía.

39. Cf. por ejemplo EN VI 9, 1142a23-24. Se pueden distinguir a grandes trazos cuatro tendencias en la historia de la interpretación de esta distinción: 1) una tendencia subraya el carácter práctico de la ф@óvๆoıs y, por oposición, la descriptividad de la ciencia práctica (J. Walter, E. Frank); 2) la tendencia opuesta subraya la unicidad del saber

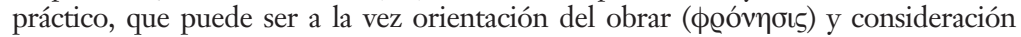
científica de las acciones (G. Teichmüller, R. A. Gauthier-J. Y. Jolif, W. F. R. Hardie); 3) una tercera tendencia atribuye un carácter tópico-dialéctico al saber práctico en su conjunto (A. Grant, J. Bumet, W. Hennis, G. Bien); 4) hay, finalmente, una última tendencia que se opone a esta atribución, argumentando que, puesto que Aristóteles niega que la dialéctica sea una ciencia, atribuir a la filosofía práctica un carácter tópico-dialéctico equivaldría a negar que la filosofía práctica fuera un saber científico (F. Susemihl, H. Kuhn, J. A. Stewart, O. Gigon, H. H. Joachim). 
Gracias a su esfuerzo por delimitar el campo epistémico de la $\pi \varrho \hat{\alpha} \xi ı \zeta$, Aristóteles llega a una diferenciación de los tipos de saber científico que no solo salva los fenómenos, sino que, además, per-

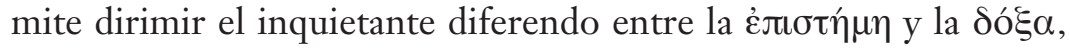
que se había bosquejado en los presocráticos (en Heráclito y en Parménides) y se había profundizado aún más en los sofistas y en

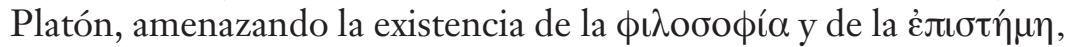
que acababan de constituirse. En efecto, la determinación de la posibilidad de una consideración epistémica en el dominio de la $\pi \varrho \hat{\alpha} \xi ı \varsigma$ no solo salva este dominio en su autonomía, sino que, además, define y delimita el dominio de la $\theta \varepsilon \omega \varrho a^{\alpha}{ }^{40}$, salvando la posibilidad de esta, amenazada por el riesgo de quedar expuesta a un conflicto con la $\delta$ ó $\xi \alpha$. Por otra parte, el carácter particular de la ciencia práctica permite a Aristóteles una suerte de rehabilitación de la $\delta$ ó $\xi \alpha$, en la medida en que, en la diferenciación de grados epistémicos de universalidad y exactitud, no hay ya ninguna razón para establecer una oposición total y radical entre la $\delta o ́ \xi \alpha$ y la $\dot{\pi} \pi \sigma \tau \eta \dot{\mu \eta}$.

Cuando en la Edad Media y, más aún, en la época moderna tenga lugar una identificación de la scientia y de la theoria, en la cual encuentra su origen el ideal del saber unitario y metódico y bajo cuya égida dicho ideal se desarrolla, se habrá perdido definitivamente la preciosa obra de diferenciación realizada por Aristóteles, aun cuando las clasificaciones escolásticas y enciclopédicas del saber hayan conservado la estructura formal de la clasificación aristotélica. De esta suerte, la comprensión "científica” del saber práctico que nos proponen los modernos — por ejemplo, Spinoza para la ética, Hobbes para la política, Pufendorf para el derecho, Petty para la economía - representa, paradójicamente, una regresión a una

40. "L'originalité d'Aristote —observaba con razón Pierre Aubenque, que, así, ha puesto en evidencia un punto fundamental - ne consiste donc pas, comme on le croit parfois, dans l'affirmation du caractère pratique de la prudence, ni d'aillieurs dans celle de son caractère intellectuel. Car Platón n'avait jamais dit autre cose de sa sagesse, qui, indifféremment appelée sophia ou phronesis, était déjà indissolublement théorique et pratique. Et tel était déjà le sens de la doctrine socratique de la vertu-science. L'originalité d'Aristote consiste, en réalité, dans une nouvelle conception des rapports de la théorie et de la pratique, conséquence elle-méme d'une rupture pour la première fois consommée dans l'univers de la théorie" (P. Aubenque, op. cit., 144; subrayado del autor). 
comprensión pre-aristotélica del saber práctico (y de la relación entre la teoría y la praxis). De ella nacen también los problemas fundamentales de la determinación moderna de las ciencias del obrar.

\section{Consideraciones conclusivas}

Precisamente en vista de estos problemas no resueltos los neoaristotélicos alemanes, a través de la "rehabilitación de la filosofía práctica", han abrevado nuevamente en Aristóteles, a decir verdad, de un modo un tanto rapsódico, y condicionados, a mi modo de ver, más por las aporías de la modernidad y por la ansiedad de resolverlas que por la exigencia de comprender efectivamente las transformaciones históricas y conceptuales que llevaron a la modernidad a retroceder más allá de la determinación aristotélica del saber práctico. En efecto, todas las intuiciones y todas las tesis programáticas sostenidas con vigor por los neo-aristotélicos alemanes están, sin duda alguna, en correspondencia con problemas y exigencias experimentados en la auto-comprensión contemporánea de las disciplinas prácticas y, en particular, con la exigencia de restituir al saber referido al obrar su capacidad de orientación. En la oposición crítica a la descriptividad, al carácter exclusivamente constatativo y neutro de la consideración del obrar desarrollada en las ciencias sociales y humanas, el paradigma por excelencia de dicho saber adecuado a la

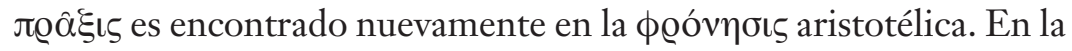
concepción aristotélica, en efecto, el saber fronético — aun cuando tiene una dignidad inferior respecto del saber teórico de la sabiduría (бoфía), en razón del grado inferior de la perfección de ser de su objeto, esto es, las acciones humanas y no las realidades divinas y celestes - tiene, sin embargo, un carácter que el saber teorético no posee, a saber, la capacidad de dirigir, de orientar la acción ${ }^{41}$.

Subrayando el carácter de orientación propio del saber práctico, los neo-aristotélicos buscan oponerse así a los nuevos intelectualismos predicados en ética y en política; buscan en dicho carácter del saber práctico un punto de apoyo para reunir en el obrar el

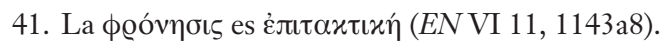


momento del conocimiento y el de la actuación, para reconciliar el "saber lo que es el bien" y el "hacer el bien". Para ello, rehabilitan también el elemento de la decisión, de la elección deliberada, de

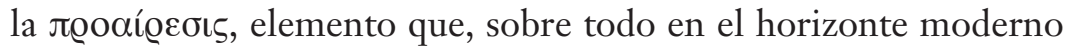
de la incomprensión teoreticista del saber práctico, fue considerado a menudo como un elemento imponderable e irracional. Unos lo rechazan y lo expulsan de sus teorías, otros lo exaltan como raíz originaria del obrar, especialmente, del obrar político. Contra ambos extremos, teoreticismo e intelectualismo, de un lado, voluntarismo y decisionismo, del otro, los neo-aristotélicos se proponen abrir el camino de una reconciliación entre razón y decisión ${ }^{42}$.

Por otra parte, esta crítica del intelectualismo ético está asociada, en ciertos neo-aristotélicos, a una crítica de la disociación entre moral y eticidad, dicho de otro modo, a una crítica de la separación, típicamente moderna, entre la racionalidad y la universalidad de los principios del obrar, por un lado, y el carácter contextual opaco, particular e históricamente condicionado de las instituciones y las costumbres concretas en las cuales el obrar concreto se efectúa y se sedimenta, por el otro. Contra esta disociación y esta separación, los neo-aristotélicos afirman la necesidad de una interpenetración de la moralidad y la eticidad, de la universalidad y las determinaciones concretas, pues solo en el carácter concreto del $\hat{\eta} \theta 0 \varsigma$, de las costumbres e instituciones, puede ser realizada la racionalidad práctica en la búsqueda del verdadero universal, es decir, el universal concreto. Por lo demás, todo esto equivale a una crítica de la utopía, dicho de otro modo, a una crítica de

42. Es significativo que quienes permanecen atados al paradigma kantiano del saber práctico hayan procurado obtener una función análoga de orientación de la facultad del juicio (Urteilskraft). Desarrollando una hipótesis propuesta originalmente por Hannah Arendt, se ha utilizado para esta función la determinación kantiana de la facultad del juicio reflexivo (reflektierende Urteilskraft), tal como ella es delimitada, en la Kritik der Urteilskraft, respecto de la del juicio determinante. Haciendo completa abstracción de la comprensión kantiana de la ética y la política, se ha intentado considerar el juicio reflexivo como el paradigma del saber adecuado a la $\pi \hat{\alpha} \xi ı$ เ . A diferencia tanto de la razón (Vernunft) en tanto facultad del pensamiento coherente, como del entendimiento (Verstand) en tanto facultad del pensamiento autónomo, la facultad de juzgar, en tanto "modalidad ampliada del pensar" (erweiterte Denkungsart), se funda en el siguiente principio: "an der Stelle jedes andern denken", y, como tal, es apta para aplicarse al dominio de la ética y de la política, como vehículo que es de una universalidad estrechamente unida al carácter concreto de las situaciones particulares. 
la moralidad y la racionalidad abstractas que creen poder anticipar, en una descripción teórica, los contenidos de la vida en la felicidad y que, aferrándose a la imagen que ellas mismas se hacen de la vida dichosa, pretenden revolucionar el $\hat{\eta} \theta$ o s y el vó $\mu$ o s existentes.

Sea como fuere, si los neo-aristotélicos han tenido algún mérito, es seguramente el de haber despertado una conciencia crítica respecto de la comprensión moderna del obrar y del saber que debe corresponderle. Aunque marcada por el carácter dudoso de la recuperación de fragmentos del aristotelismo, de su utilización en contextos heterogéneos, y por la rehabilitación de modelos de racionalidad práctica como la prudencia, la reasunción neo-aristotélica de la filosofía práctica ha llamado nuevamente nuestra atención sobre una comprensión del obrar y del saber relacionado con él, que, aunque antigua, conserva toda su validez. En la autorepresentación cultural de la época contemporánea, en su confusión tras la crisis del pensamiento dialéctico y atormentada por los vientos furiosos del pensamiento antidialéctico, la rehabilitación de la filosofía práctica ha abierto una perspectiva a partir de la cual es de nuevo posible considerar de manera crítica la identificación de la ciencia y la teoría llevada a cabo en la modernidad así como la idea paleo-moderna y moderna de razón y su desarrollo en el sentido de la mera instrumentalidad y la mera funcionalidad.

Seguramente, muchos problemas permanecen abiertos. Quisiera limitarme a indicar brevemente el que me parece más inquietante. El saber fronético, como se vio, es rehabilitado por los neoaristotélicos en el marco general de una tentativa por responder a la crisis de la idea moderna de razón, privada de toda sustancialidad e incapaz de indicar de modo vinculante fines últimos al obrar del hombre. Se lo ha invocado, pues, para ayudarnos en el marco de un

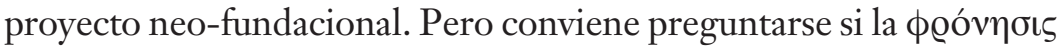
no sería, en Aristóteles, un saber que concierne a los medios aptos para alcanzar un fin y no al fin mismo. Y, si lo que le falta al mundo moderno, en toda su "imprudencia", no son precisamente los medios, que la ciencia pone, efectivamente, cada vez más a disposición, ¿cómo se puede esperar obtener indicaciones sobre los fines a través

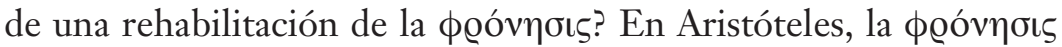
podría garantizar el equilibrio entre la eficacia de los medios y la 
cualidad moral de los fines y con ello, finalmente, el éxito del obrar, porque estaba pensada en el marco específico trazado por la ciencia práctica y en el marco más general de su antropología, de su cosmología y de su metafísica, y, por ello, era capaz de utilizar los conceptos operativo extraídos de dichos marcos. En los neo-aristotélicos,

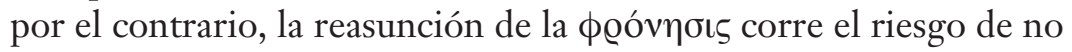
alcanzar su objetivo, pues aquí el saber fronético está concebido en un horizonte post-metafísico, en un horizonte demasiado chato y más débil que el de Aristóteles, dicho de otro modo, en un horizonte mínimo o incluso en la ausencia de todo marco, de todo hito, por referencia al cual el saber fronético pudiera orientarse. En una

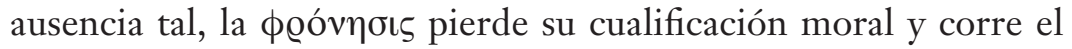

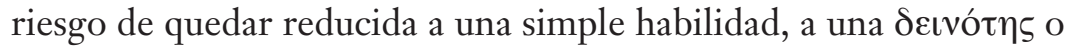

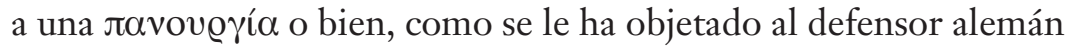

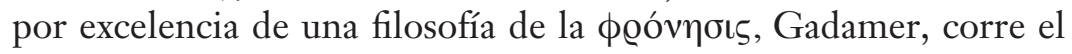
riesgo de convertirse en una ideología, especialmente — como se ha podido sostener - la ideología de un agradable relativismo cultural moderado de tipo conservador.

Justamente a causa de esta situación de desorientación del mundo contemporáneo, a consecuencia de la abstinencia forzada que ha engendrado lo que yo llamaría una situación de destierro post-metafísico, una pregunta que nos constriñe exige de nosotros una respuesta. Esta pregunta, para parafrasear un célebre título kantiano, podría ser formulada del siguiente modo: ¿qué significa orientarse en el obrar? Nadie, ni los neo-aristotélicos ni tampoco sus adversarios ni ningún otro, puede afirmar hoy en día tener una respuesta a esta pregunta. ¿'Tienen acaso una estrategia para controlar a situación? Tal vez. ¿¿Una ayuda para remediar la crisis? Probablemente también. Pero cuanto más se controla, cuanto más se remedia, más aguda se vuelve la sensación de destierro. Por ello, incluso a través de múltiples unilateralidades y forzamientos, las consideraciones y los problemas que la rehabilitación de la filosofía práctica y los neo-aristotélicos han propuesto a nuestra atención permanecen en su inquietante actualidad. 